Original article

\title{
Evaluation of cone-beam computed tomography over a small field of view in a water bath based on the modulation transfer function with repeating-edge oversampling
}

\author{
Motohiro Ito $^{1)}$, Yusuke Hayashi ${ }^{1)}$, Kunihito Matsumoto ${ }^{1,2)}$, Yoshinori Arai ${ }^{1,2)}$, and Kazuya Honda ${ }^{1,2)}$ \\ Department of Oral and Maxillofacial Radiology, Nihon University School of Dentistry, Tokyo, Japan \\ ${ }^{2)}$ Division of Advanced Dental Treatment, Dental Research Center, Nihon University School of Dentistry, Tokyo, Japan
}

(Received September 15, 2020; Accepted November 11, 2020)

\begin{abstract}
Purpose: The spatial resolution of cone-beam computed tomography (CBCT) in small fields of view (FOVs) is important for clinical applications. However, it is difficult to measure spatial resolution reliably due to error factors such as noise. The aim of this study was to obtain a modulation transfer function (MTF) more accurately.

Methods: A CBCT apparatus was used with small FOV. An aluminum pipe slightly tilted at an inclination ratio of 77/3 (25.7) was used as the measurement phantom. The MTF was calculated from the edge image of the phantom. The actual oversampling ratio was determined by regression analysis. The experiment was repeated 16 times and the edge-spread function (ESF) was approximated by the least-square method. Furthermore, a low-pass filter (LPF) was applied to eliminate the component at frequencies above the Nyquist frequency. Finally, the MTF was calculated from the pre-processed ESF.

Results: Results showed that pre-processing reduced the noise of the ESF. The MTFs at frequencies of 1.0 and $2.0 \mathrm{LP} / \mathrm{mm}$ were 0.59 and 0.18 , respectively, in air and 0.52 and 0.16 , respectively, in water.

Conclusion: The repeating-edge oversampling method combined with ESF pre-processing improved the accuracy of the MTF under clinically relevant conditions with a phantom.
\end{abstract}

Keywords; computed tomography, cone beam, dental, modulation transfer function

\section{Introduction}

Cone-beam computed tomography (CBCT) for small fields of view (FOVs) was developed by Arai et al. [1] in the late 1990s. The technique can be carried out with a low exposure dose and provides high resolution (Directorate-General for Energy [European Commission], Cone beam CT for dental and maxillofacial radiology, 99-110, Publications Office of the European Union, 2012) [2,3]. The most important factor in CBCT image evaluation is the spatial resolution, which has been studied extensively [1,4-16]. Proposed approaches include subjective methods and methods based on the modulation transfer function (MTF) using fast Fourier transfer (FFT) [1,4-7,9-16]. However, none of these approaches offer exceptionally reliable results [11]. Additionally, there are some discrepancies between the results of subjective studies and those based on the MTF [11]. Many of the measurements were conducted in air, which is effective for quality control of the CBCT apparatus $[1,4,6,7,14]$, but is not consistent with clinical imaging conditions. Additionally, previous studies used a lower tube current and tube voltage compared with clinical use conditions in air. Moreover, the studies did not consider radiation scattering that is often caused by soft tissue.

To measure the MTF under clinically relevant conditions, it is necessary to use a water bath or a polymethyl methacrylate (PMMA) equivalent

Correspondence to Dr. Yoshinori Arai, Department of Oral and Maxillofacial Radiology, Nihon University School of Dentistry, 1-8-13 Kanda-Surugadai, Chiyoda-ku, Tokyo 101-8310, Japan Fax.+81-3-3219-8354 E-mail: arai.yoshinori@nihon-u.ac.jp

J-STAGE Advance Publication: December 14, 2020

Color figures can be viewed in the online issue at J-STAGE.

doi.org/10.2334/josnusd.20-0479

DN/JST.JSTAGE/josnusd/20-0479 to soft tissue $[5,8,10]$ and to set tube current and tube voltage to those used in clinical settings. However, the image intensity acquired with $\mathrm{CBCT}$ in a small FOV is not stable, so the CT values cannot be determined accurately. Furthermore, the $\mathrm{CBCT}$ image contains significant noise because of the low radiation dose [2,3]. Therefore, the MTF varied significantly and exhibited low reproducibility in previous studies [11,15]. Additionally, it is theoretically impossible to calculate the MTF accurately because of scattering noise [15]. Therefore, MTF results may be unreliable in clinical settings. Some studies revealed that MTF results are not obtained even if the measurement is conducted as described in the operation manual [15].

Generally, the MTF is calculated by applying the FFT to the line spread function (LSF) obtained from a slit or wire image. Therefore, the LSF would ideally have no noise to obtain an accurate MTF. Because there is little noise when imaging in air, errors in the MTF can be minimized. However, images taken in a water bath or for clinical applications have significant scattering noise [17], making it difficult to calculate the MTF accurately. If the noise increases, the error of MTF also increases $[18,19]$. Therefore, it is necessary to reduce this noise before calculating the MTF.

The purpose of this study was to obtain an accurate MTF by using repeating-edge oversampling in water, which is comparable to a clinical imaging scenario. The edge method $[4,5,11-13,16,20]$ offers higher contrast and is more resistant to noise than the wire method. In this study, a pipe of a rotating body was used as a subject for obtaining an edge image [4]. For the oversampling method, the pipe was slightly tilted $[11,16,20]$, and edge-spread functions (ESFs) were acquired by repeatedly imaging, synthesizing the data, and applying low pass filter (LPF) [18]. Here, the authors demonstrate the ability of this noise-reduction method to improve the accuracy of the MTF in water as a clinically relevant condition.

\section{Materials and Methods}

\section{Measurement phantom}

The parameters of the measurement phantom are shown in Table 1 and Fig. 1. An aluminum pipe (A1050TD, AQR Co., Tokyo, Japan) with a diameter of $10 \mathrm{~mm}$ and thickness of $1 \mathrm{~mm}$ was used as an object. The aluminum pipe was installed at a slight tilt relative to the vertical direction using an original pedestal. The pedestal comprised a base plate (diameter: $50 \mathrm{~mm}$ ), a main pipe (outer diameter, $30 \mathrm{~mm}$; height, $80 \mathrm{~mm}$; thickness, 1 $\mathrm{mm}$ ), and a square bolster (length of each side, $3 \mathrm{~mm}$; Table 1). The main pipe, which was commercially available, was cut at a right angle to the longitudinal direction. The spirit level was fixed on the top of the main pipe. The aluminum pipe was tilted $2.23^{\circ}$, so the theoretical inclination ratio was $77: 3$ (25.7). Thus, the theoretical oversampling ratio was 25.7 .

\section{Image acquisition}

Veraview X800 (J. Morita MFG. Corp., Kyoto, Japan) was used as the CBCT apparatus for the experiments. The exposure conditions were the same as those typically used in clinical settings: the tube voltage was 100 $\mathrm{kV}$, the tube current was $5 \mathrm{~mA}$, the exposure time was $17 \mathrm{~s}$, the rotation angle was $360^{\circ}$, the FOV was $40 \mathrm{~mm}$ in diameter $\times 40 \mathrm{~mm}$ in height, and the voxel size was $0.125 \mathrm{~mm}$ (isotropic). The number of voxels was 320 in diameter $\times 320$ in height. The water bath was $180 \mathrm{~mm}$ in diameter and 100 $\mathrm{mm}$ in height. The measurement phantom was positioned in the center of the FOV using the laser beam of the CBCT apparatus. The pedestal was adjusted such that its horizontal plane was at the spirit level. The direction of the inclination of the aluminum phantom pipe coincided with the mid- 
A

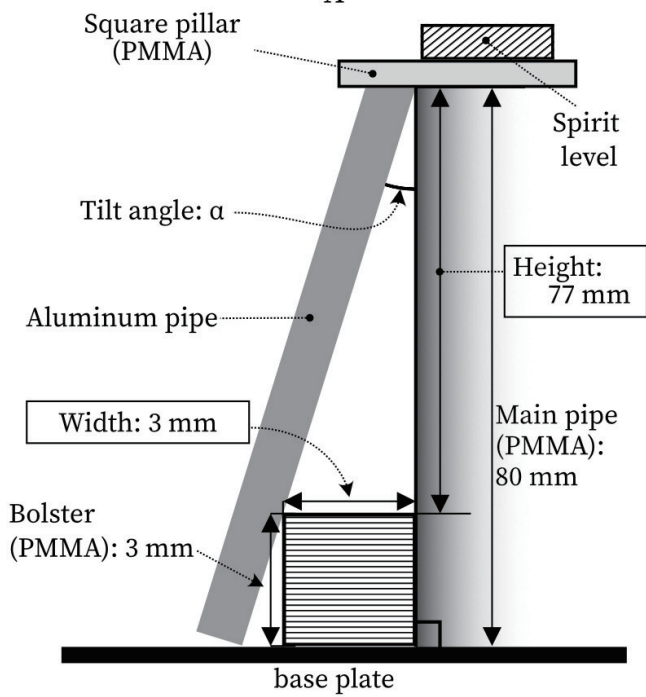

Theoretical inclination ratio: $77 / 3$ (25.7)

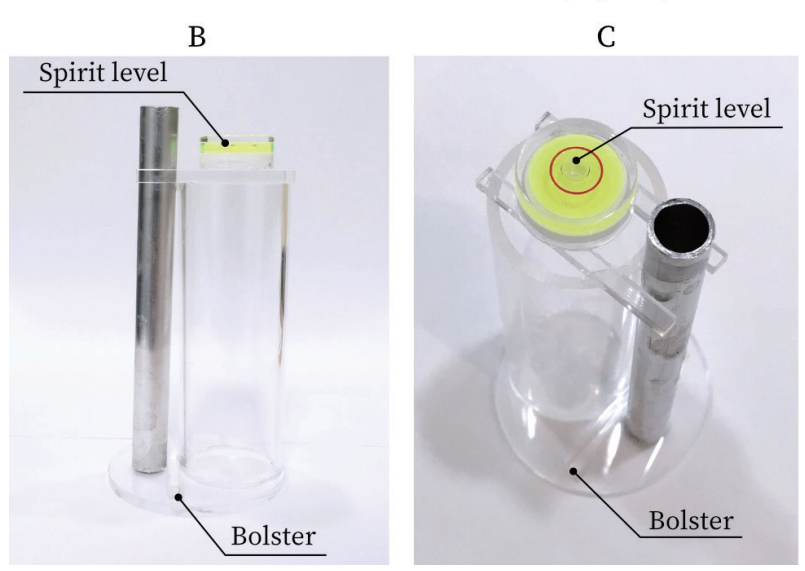

Fig. 1 The measurement phantom. The aluminum pipe was tilted, and the theoretical inclination ratio was 77:3 (25.7). A spirit level of polymethyl methacrylate (PMMA) was set on the main pipe. Design of the pedestal (A) and photographs from a side view (B) and diagonal view (C)

Table 1 Measurement phantom characteristics

\begin{tabular}{ll}
\hline Parts & Geometry \\
\hline Aluminum pipe (diameter $\times$ thickness in $\mathrm{mm})$ & $10 \times 1$ \\
Base plate (PMMA) (diameter in mm) & 50 \\
Main pipe (PMMA) (diameter $\times$ length $\times$ thickness in mm) & $30 \times 80 \times 1$ \\
Bolster (PMMA) (edge length in mm) & 3 \\
Spirit level (diameter $\times$ height in mm) & $20 \times 8$ \\
\hline The inclination ratio was defined as 77:3 (25.7). PMMA, polymethyl methacrylate
\end{tabular}

sagittal plane.

First, images were acquired in air. A copper plate (thickness: $1.0 \mathrm{~mm}$ ) was installed in front of the X-ray tube to attenuate the X-ray irradiation to a level of that in water. Image acquisitions were carried out 16 times. The CBCT apparatus was reset to the home position after each acquisition. Each exposure was carried out at equal intervals $(5 \mathrm{~min})$. The image reconstruction kernel, G_003 + H_205 for dental, was applied to the acquired data with i-Dixel software (J. Morita MFG. Corp.).

Next, images were acquired in water. A water bath was filled with degassed water to avoid air bubbles, which attach to the aluminum pipe (Fig. 2). The copper plate that was placed in front of the X-ray tube for air imaging was removed for imaging in water. Image acquisition in water was repeated 16 times under the same exposure conditions as in air.

\section{Image analysis}

\section{Data acquisition}

A continuous series of 320 axial CT images of the phantom were obtained and exported as 16-bits grayscale DICOM (digital imaging and communications in medicine) format images using i-Dixel software. Image analysis was carried out with custom software written in Python (https://www.

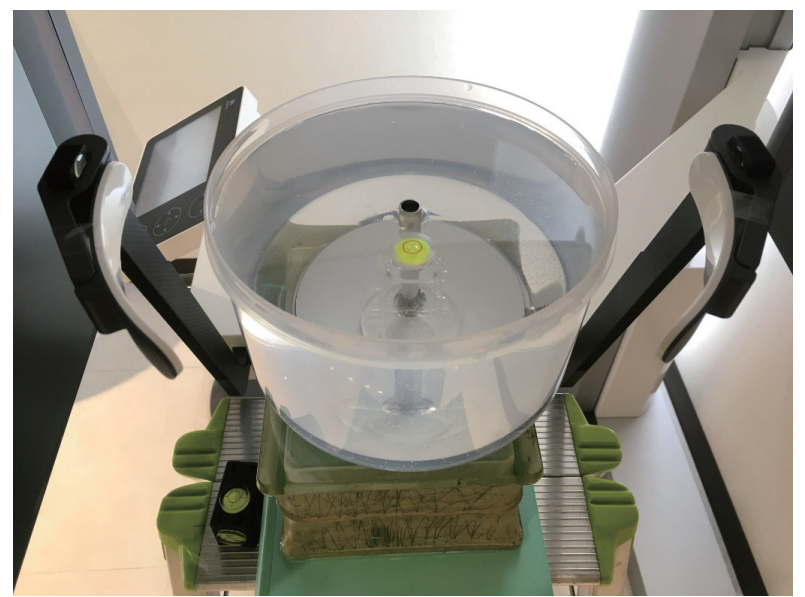

Fig. 2 The water bath. The measurement phantom was submerged in the center of the water bath. The field of view was centered on the center of the phantom.

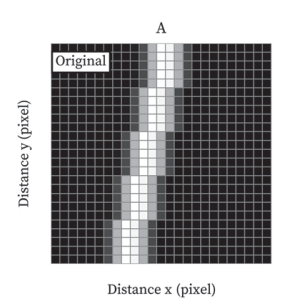

Distance $x$ (pixel)

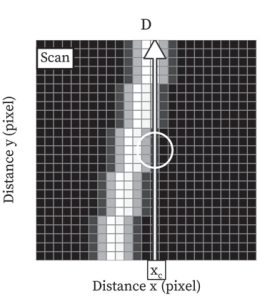

Fig. 3 Schema of the edge spread function (ESF) measurement process. Center of a sagittal section of the aluminum pipe (A). Differentiated image of a (B). The peak positions are shown as white pixels. The line derived from a regression analysis is shown as a black striped arrow on the white pixels. The center of the regression line, $x_{c}$, was obtained $(C)$. The ESF was scanned along the $y$-axis through $x_{c}(D)$. The center of the ESF, $y_{c}$, was obtained from the center of the intensity of the ESF, $\mathrm{i}_{\mathrm{c}}(\mathrm{E}$, black circle).

python.org/) with scientific computing library NumPy (https://numpy. org/). The obtained axial slices were imported as $3 \mathrm{D}$ volume data.

\section{Estimating the oversampling rate and center of the regression line}

The mid-sagittal section of the phantom was reconstructed from the $3 \mathrm{D}$ volume data (Fig. 3A). The region of interest (ROI) was set on the front side of the aluminum pipe (Fig. 3A). This image was differentiated in the lateral direction (x-axis) to emphasize the edges (Fig. 3B). The positions of the maximum intensity values were identified as the edge positions from the lateral direction (x-axis) and displayed as white pixels (Fig. 3C). The regression line was shown as a black striped arrow on the white pixels. The actual oversampling ratio was equivalent to the inclination ratio of the regression line. The center of the regression line, $\mathrm{x}_{\mathrm{c}}$, was obtained as the center of the ESF (Fig. 3C, white circle).

\section{Acquiring and aligning the ESF by the least-square method}

The ESF was acquired as a straight line through $\mathrm{x}_{\mathrm{c}}$ along the $\mathrm{y}$-axis (Fig. 3D white arrow), and the resulting ESF intensities were shown in Fig. 3E. This process was repeated 16 times for each image acquisition, so $16 \mathrm{ESFs}$ were obtained. Then, the center of the profile on each ESF was calculated as follows: first, the moving average of the ESF was calculated using a window width equal to the oversampling ratio. Then, the mid-intensity position, $\mathrm{i}_{\mathrm{c}}$, of each ESF at $\mathrm{x}_{\mathrm{c}}$ was estimated (Fig. 3E, black circle), and the center of the ESF position, $y_{c}$, was obtained from $i_{c}$ (Fig. 3E). The 16 ESFs were overlaid and aligned such that their $y_{c}$ points were aligned by the least-square method then averaged to obtain $\mathrm{ESF}_{\text {ave }}$. 


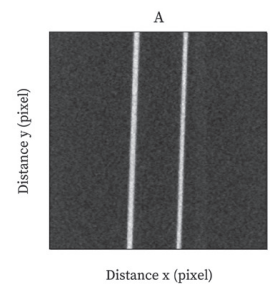

Distance $x$ (pixel)
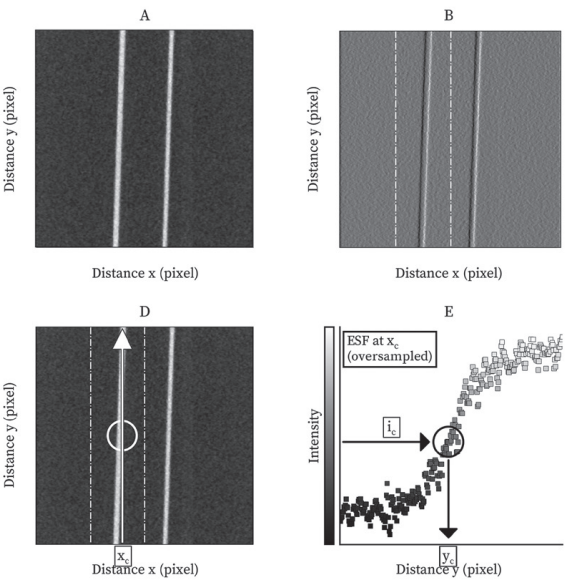

Distance $x$ (pixel)
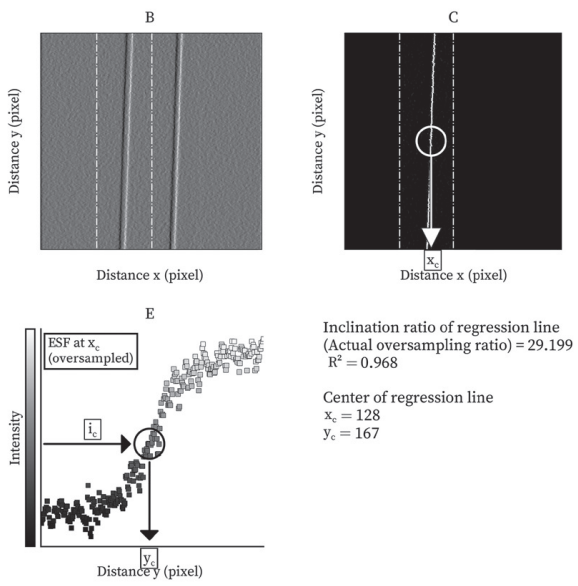
(Actual oversampling ratio) $=29.199$
$\mathrm{R}^{2}=0.968$ Center of regression line $\mathrm{x}_{\mathrm{c}}=128$
$\mathrm{y}_{\mathrm{c}}=167$
Inclination ratio of regression line
(Actual oversampling ratio) $=29.199$

Fig. 4 Actual ESF measurement process in water for one example. Center of the sagittal section of the aluminum pipe phantom (A). Differentiated image of a (B). The peak positions are shown as white pixels. The regression analysis was performed, and the center of the regression line as, $\mathrm{x}_{\mathrm{c}}$, was obtained. The white circle represents the center of the regression line (C). The ESF was scanned along $y$-axis along the center line from $\mathrm{x}_{\mathrm{c}}(\mathrm{D})$. The position of the center of the ESF was obtained as $\mathrm{y}_{\mathrm{c}}(\mathrm{E}$, black circle).

Table 2 Oversampling ratios (OSR)

\begin{tabular}{lll}
\hline Condition & OSR & $\mathrm{R}^{2}$ \\
\hline Theoretical & 25.7 & - \\
Air (actual) & 27.2 & 0.988 \\
Water (actual) & 29.8 & 0.969 \\
\hline
\end{tabular}

\section{Filtering on the Nyquist frequency}

The frequency components of $\mathrm{ESF}_{\text {ave }}$ above the Nyquist frequency [21] were removed by applying a low-pass filter (LPF). The moving-average method was used as the LPF, and the window width was equal to the oversampling ratio.

\section{Calculation of the MTF from ESF}

The LSF was obtained by differentiating $\mathrm{ESF}_{\text {ave. }}$. The LSF was extrapolated up to 65536 (216) pixels by padding it with zeros, then the FFT was applied. Finally, the MTF was calculated from the FFT of the LSF as described previously [20].

\section{Statistical processing and Comparison of MTF}

Statistical processing was conducted with Excel 2013 (Microsoft Corp., Redmond, WA, USA) and SPSS Statistics for Windows version 25.0 (IBM Corp., Armonk, NY, USA). The arithmetic mean, standard deviation (SD) of the actual oversampling ratio, and coefficient of determination $\left(\mathrm{R}^{2}\right)$ of the regression line were calculated. The maximum of the deviation and SD of the distance of the ESF center position from the y-axis was used as $y_{c}$ and an $F$-test was performed. The 16 MTFs obtained independently in air and in water were used to obtain the synthesized MTFs for air and water, respectively; the MTFs were then used to derive $\mathrm{ESF}_{\text {ave }}$. The average and SD of the MTFs at frequencies of 1.0 and 2.0 line pairs $/ \mathrm{mm}(\mathrm{LP} / \mathrm{mm})$ were calculated and compared using Welch's $t$-test. The statistical significance level was set at $P<0.05$.

\section{Results}

The analysis of one of the images captured in the water bath is shown in Fig. 4. Figure 4A shows the mid-sagittal image of aluminum pipe phantom. The differentiated image is shown in Fig. 4B. The estimated positions of maximum intensity values are shown as white pixels, and the center of the calculated regression line $\mathrm{x}_{\mathrm{c}}$ is shown as a white arrow in Fig. 4C. The actual inclination ratio was 29.199. The center of line $x_{c}$ was 128. The ESF was obtained by scanning along line $\mathrm{x}_{\mathrm{c}}$ (Fig. 4D); the result is shown in Fig. 4E. Additionally, $\mathrm{y}_{\mathrm{c}}$ was 167 (Fig. 4E, black circle).

As shown in Table 2, the theoretical oversampling ratio was designed as 25.7 , but the actual value in air was 27.2 , while that in the water bath was 29.8 (arithmetic means), both of which were higher than the theoretical
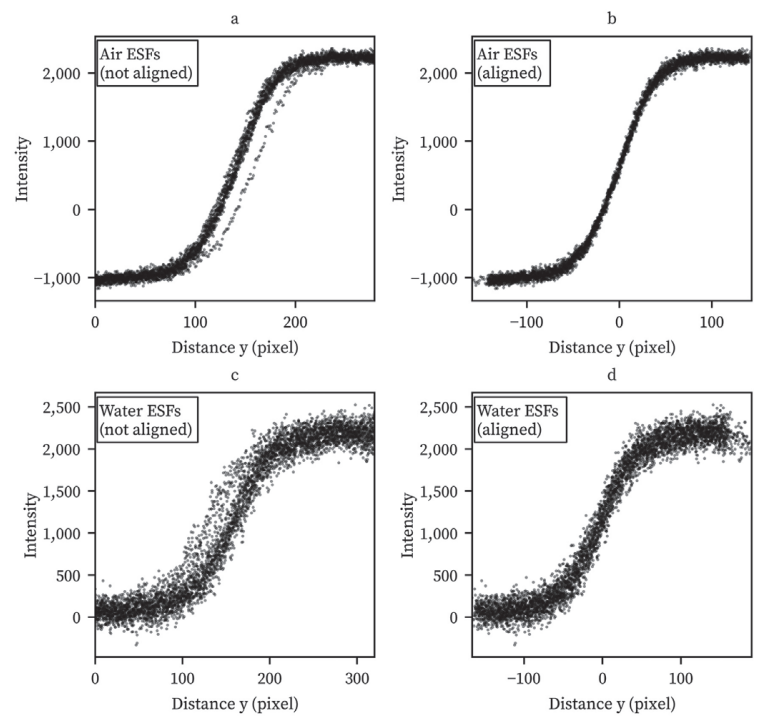

Fig. 5 Alignment of individual ESFs taken 16 times in air before alignment (a). After alignment in the air, the ESF was shifted up by 17 pixels (b). The 16 ESFs in water were obtained before alignment (c). After alignment in water, the ESF was shifted up to 25 pixels (d).

Table 3 ESF shift ranges $\left(y_{c}\right)$

\begin{tabular}{cll}
\hline Condition & Maximum & Standard deviation \\
\hline Air & 17.0 & $5.072 *$ \\
Water & 25.75 & $11.852^{*}$ \\
\hline *A statistically significant difference based on an $F$-test $(P<0.05)$. &
\end{tabular}

value. The coefficient of determination, $\mathrm{R}^{2}$, for the regression analysis was 0.988 in air and 0.969 in water.

Figure 5A shows the 16 ESFs in air before alignment, and Fig. 5B shows the same ESFs after alignment. The maximum of the deviation of the ESF center position as $\mathrm{y}_{\mathrm{c}}$ was 17 (SD 5.1) in air. Figure 5C and D show the ESF in water before and after alignment, respectively. The maxima of the deviation and SD of $\mathrm{y}_{\mathrm{c}}$ were 25.8 and 11.8, respectively. There was a statistically significant difference between the SD values obtained in air and water based on an $F$-test (Table 3 ).

Figure 6 shows the $\mathrm{ESF}_{\text {ave }}$, LPF applied to the $\mathrm{ESF}_{\text {ave }}$, and LSF; Fig. 6A, $\mathrm{B}$, and $\mathrm{C}$ show those in air, respectively, and Fig. $6 \mathrm{D}, \mathrm{E}$, and $\mathrm{F}$ show those in water, respectively. Figure 7 shows the 16 individual MTFs obtained in air and water. Figure 8 shows the synthesized MTF obtained in air and water. The MTFs at $1.0 \mathrm{LP} / \mathrm{mm}$ were 0.59 in air and 0.52 in water, and the difference was statistically significant. The MTFs at $2.0 \mathrm{LP} / \mathrm{mm}$ were 0.18 in air and 0.15 in water; this difference was not statistically significant (Table 4).

\section{Discussion}

In image quality evaluation, the spatial resolution is especially important in combination with noise evaluation $[11,14]$. CBCT in a small FOV enables the observation of the fine morphology of anatomy such as the root canal $[2,22]$. The spatial resolution can be evaluated using subjective methods [9] and objective methods based on physical measurements [11]. The subjective method is relatively simple, but it requires a special phantom. In the objective method, a measurement phantom is generally imaged to obtain the point spread function (PSF) or LSF applying the FFT to calculate the MTF $[16,20]$.

The slit method is used to obtain the LSF for two-dimensional films. This technique is based on the property that a pulse contains all frequency components. For three-dimensional imaging, the wire method is used. This approach is an expansion of the slit method. To obtain accurate measurements, the wires need to be very thin, ideally smaller than the voxel size. For example, Watanabe et al. used a tungsten wire with a diameter of 0.1 $\mathrm{mm}$, which was thinner than the voxel size of $0.125 \mathrm{~mm}[6,7]$. Other wire phantoms for CBCT were $0.25 \mathrm{~mm}$ in diameter $[8,10,15]$, but they are not suitable for measuring the MTF in high-resolution CBCT because they are wider than the voxel size. The thinner the wire is, the closer it is to 

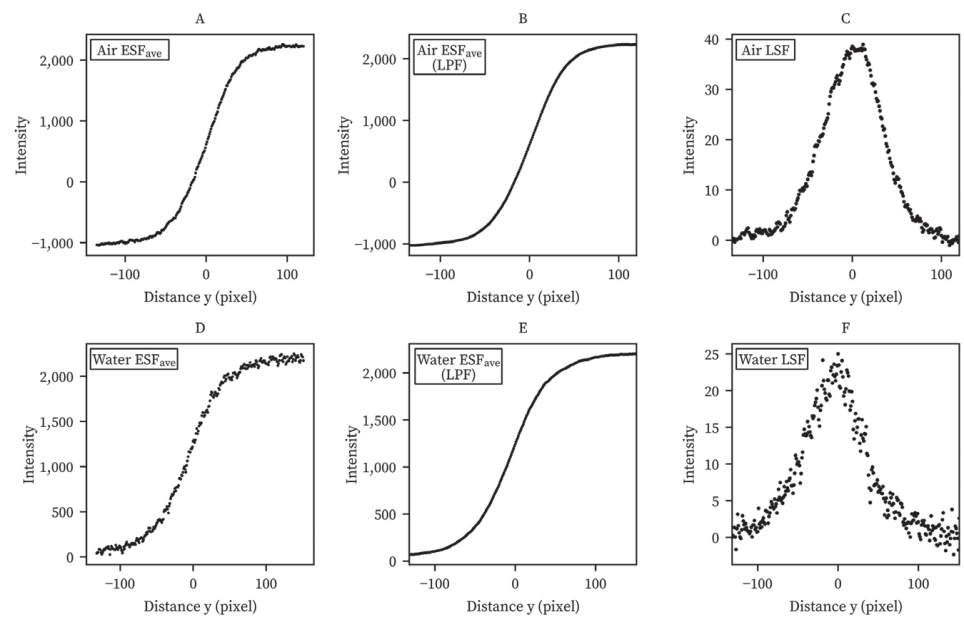

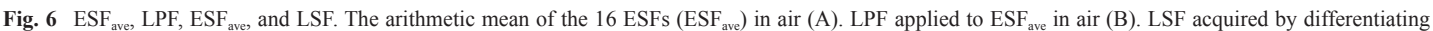
the filtered $\mathrm{ESF}_{\text {ave }}(\mathrm{C})$. Arithmetic mean of $16 \mathrm{ESFs}$ in water (D). LPF applied to $\mathrm{ESF}_{\text {ave }}$ in water $(\mathrm{E})$. The LSF acquired by differentiating the filtered $\mathrm{ESF}_{\text {ave }}(\mathrm{F})$
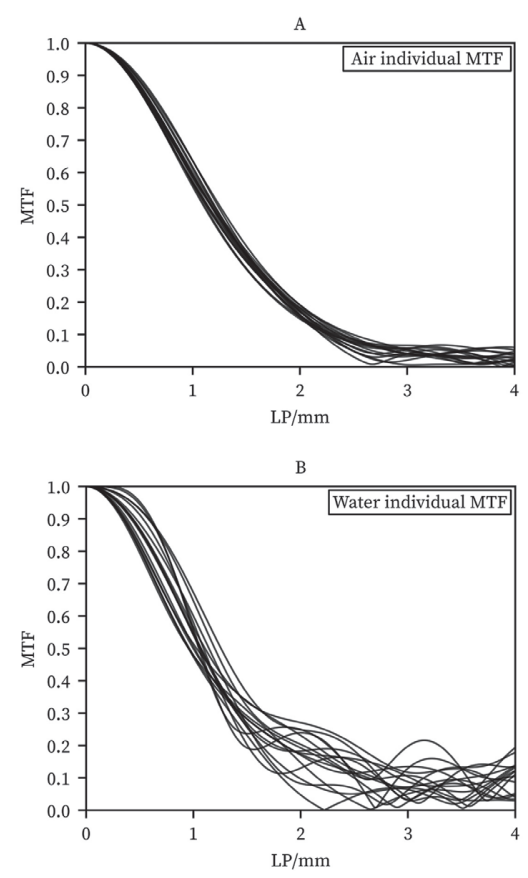

Fig. 7 Sixteen individual MTFs in air and a water bath. The MTFs in air were stable (A). However, the MTFs in the water bath were relatively unstable, especially those obtained at frequencies higher than $2.0 \mathrm{LP} / \mathrm{mm}(\mathrm{B})$.

the theoretical pulse, but thinner wires also lead to poor image contrast. For this reason, these wires cannot be detected under noisy conditions, so there is a limit to how thin the wires can be. Thus, the wire method was sensitive to noise.

In this study, the edge method was used to solve this problem. For two-dimensional imaging, radiopaque plates can be used [20]. In threedimensional imaging, a two-dimensional plate [16] or a rotating cylinder [4] can be used. The advantage of the edge method is that it results in higher contrast than the wire method, which makes it relatively resistant to noise. In the edge method, an LSF that is equivalent to that of a thin wire is obtained by differentiating the image to emphasize the edges. The limitations of clinical conditions for CBCT in small FOVs can be replicated by using a water bath, which causes X-ray attenuation and scattering [17]; these factors act as error sources in the MTF measurements. When there is a high level of noise, the obtained MTF is higher because the noise frequency components overlap [19]. To avoid the effect, the edge method was adopted in this study instead of the wire method.

In a preliminary experiment of this study, contrast to noise ratio (CNR) in air and water was measured. Consequently, CNR of in water was 3.21 times higher than in water. Assuming that the CNR in water has properties that approximate those of CNR in air, an additive average of 10.32 times, which is the square of 3.21 , is required as a minimum. Noise is reduced

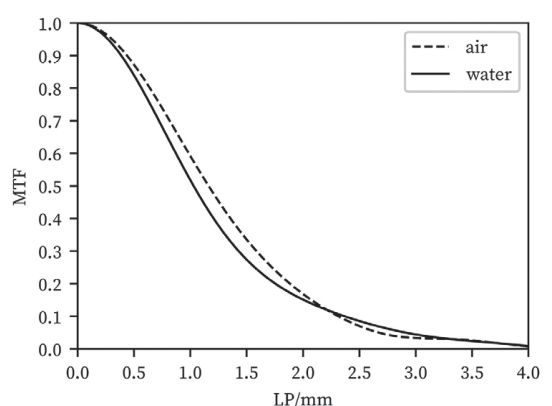

Fig. 8 Synthesized MTFs of air and water. At frequencies lower than $2.0 \mathrm{LP} / \mathrm{mm}$, the MTF in the water bath was lower than that of in air.

Table 4 Synthesized MTF results

\begin{tabular}{lll}
\hline Condition & $1.0 \mathrm{LP} / \mathrm{mm}$ & $2.0 \mathrm{LP} / \mathrm{mm}$ \\
\hline Air & $0.59\left(\mathrm{SD} 0.020^{*}\right)$ & $0.18(\mathrm{SD} 0.015)$ \\
Water & $0.52\left(\mathrm{SD} 0.058^{*}\right)$ & $0.15(\mathrm{SD} 0.064)$ \\
\hline *A statistically significant difference based on a $t$-test $(P<0.05)$. &
\end{tabular}

by half when the number of additions is quadrupled (https://terpconnect. umd.edu/ toh/spectrum/SignalsAndNoise.html\#EnsembleAveraging). Therefore, the number of additions in this study was selected from 4, 16 or 64 times. Finally, 16 times was chosen due to, which was nearest the 10.32 times.

In this study, an aluminum pipe was used because its average atomic number and density are similar to those of enamel and dentin. The purpose of this study was measurement of MTF under clinical conditions. Therefore, aluminum was selected. The aluminum pipes are standardized by JIS $\mathrm{H} 4080$. The purity is $99.5 \%$ or more, and the thickness error is $0.18 \mathrm{~mm}$ or less.

Normally, the measurement in air is taken due to quality control. The low tube voltage and current were used to prevent saturation of flat panel detector (FPD). In this study, the condition used was similar to clinical condition in water and in air. In this case, FPD was saturated in the air. For that reason, to prevent the saturation, a copper plate having a thickness equivalent to that of absorption of X-ray in water was employed for preventing saturation of FPD.

In digital images, any component that has a frequency higher than the Nyquist frequency [21] will cause error in the MTF according to the sampling theorem. The oversampling method solves this issue [20]. The oversampling method is also effective in suppressing noise [23]. The edge oversampling method relies on a slightly tilted edge. The intensity of the ESF is obtained in a direction rotated by 90 degrees with respect to the measurement direction, and the recommended angle of inclination is around 2 degrees [20]. The ratio of the base to the height of the triangle formed by this inclination is equal to the oversampling ratio. The determination of this angle is extremely sensitive, as a shift of 1 degree would cause $33 \%-50 \%$ error in the MTF. Therefore, this angle must be determined accurately. 
In a study by Watanabe et al. [6], the wire was inclined, regression analysis was performed, and the actual oversampling ratio was calculated. However, experiments with measurement phantoms often report errors that make it impossible to calculate the MTF [15]. Inaccurate measurement of the oversampling ratio is one of the reasons why the oversampling ratio cannot be determined correctly. In the present study, the pipe inclination ratio was determined to be $77: 3$ (25.7) by adding a spirit level to detect the horizontal plane, resulting in very low error in the inclination angle.

The height of FOV was $40 \mathrm{~mm}$ and a $1 \mathrm{~mm}$ thick aluminum pipe was used. Under this condition, the theoretical maximum over sampling ratio was 40:1. For reproducibility, standardized pipe with a length of $80 \mathrm{~mm}$ and standardized square bars at $1 \mathrm{~mm}$ intervals were used for the pedestal. The theoretical oversampling ratio of 40:1 is equivalent to a bolster of $2 \mathrm{~mm}$ and the height of the pedestal of $80 \mathrm{~mm}$. As a result, when a 2 $\mathrm{mm}$ square bar was used for an $80 \mathrm{~mm}$ pipe, the theoretical oversampling ratio was 78:2 (39.0), and for MTF measurement the height of $39 \mathrm{~mm}$ was required. Considering the error, it is not practical to use a length close to the height of the FOV. Therefore, the square bar was changed from $2 \mathrm{~mm}$ to $3 \mathrm{~mm}$ and the oversampling ratio was set to $77: 3$ (25.7). In this case, a height of $25.7 \mathrm{~mm}$ is required for the measurement. At this height, it was able to fit into the height of FOV $40 \mathrm{~mm}$ effortlessly.

Furthermore, the actual oversampling ratio was computed from the image of the aluminum pipe (Fig. 4A) by differentiating the intensity values (Fig. 4B), locating the points of maximum intensity value on the $\mathrm{x}$-axis (Fig. 4C), and performing regression analysis as described by Watanabe et al. [6]. The actual oversampling ratio was 10-20\% larger than the theoretical ratio of 25.7. This finding implies that the MTF had an error of $10-20 \%$ when the theoretical oversampling ratio is used relative to that when the actual oversampling ratio is used. The coefficient of determination in the regression analysis was 0.969 in the water bath and 0.988 in air, both of which were extremely high and reliable.

Therefore, the authors attempted to reduce the noise by acquiring multiple ESFs and averaging them. In this study, the 16 ESFs were overlaid [18]. However, if the obtained ESFs were simply overlaid, there would be a positional shift in the lateral direction (Fig. 5A, C), so synthesizing the ESF by simply averaging them would result in a dull waveform. To avoid this potential issue, the authors calculated the center position of each ESF as $y_{c}$ and aligned the $\mathrm{y}_{\mathrm{c}}$ of each ESF by the least-square method. This alignment made it possible to reduce the noise without bluntness, because the waveform converged even when simple averaging was performed (Fig. 5B, D). The maximum width of the lateral displacement was 25 pixels in the water bath and 17 pixels in air; converting these values such that the size of each pixel was equal to the oversampling rate, the width of displacement was 0.86 pixels $(25 / 29)$ in water, 0.62 pixels $(17 / 27)$ in air. These values did not exceed the oversampling ratio (i.e. one pixel). After this correction, $\mathrm{ESF}_{\text {ave }}$ was computed by averaging the ESFs (Fig. 5B, D). Thus, the ESF alignment avoids bluntness and error in the MTF, thus improving the accuracy of the MTF.

Finally, the oversampling method resulted in components with frequencies above the Nyquist frequency because of the noise, which caused error in the MTF. An LPF was applied to reduce this noise (Fig. 6B, E). This LPF was derived by the moving average method with a window width matching the oversampling ratio (i.e. one pixel in real space). If the window width increased, the noise was reduced $[18,23]$. In this study, the window width of 29 pixels was used to reduce the noise in the water bath. This approach makes it possible to obtain ESF curves with very low noise even in a water bath (Fig. 6B). However, the LSF in water was still noisier than that in the air. At frequencies higher than $2.0 \mathrm{LP} / \mathrm{mm}$, the relationship between the air and water MTFs was partially reversed. In this region, MTFs were as low as $10 \%$ or less, which was considered unreliable because of the effect of noise. Measurements of MTF at frequencies higher than $2.0 \mathrm{LP} / \mathrm{mm}$ in water were not considered to be accurate. According to past research, simulations also showed that noise increased the MTF at a high frequency range [19]. This is consistent with the results of this experiment. Thus, improving accuracy in this area is a subject for future research.

This is presumed to be the effect of noise. In future studies, the noise power spectrum should be checked in the water, and it will be necessary to add an optimal reconstruction function for noise and spatial resolution.

This measuring method requires 80 minutes for capturing images 16 times. For practical use, it is necessary to reduce the number of repetitions to shorten the measurement time.

In this study, the MTF of a CBCT over small FOV in a water bath was analyzed more stably using the repeating-edge oversampling method. A slightly tilted aluminum pipe was used as the phantom with a standardized inclination ratio. The actual oversampling rate was calculated using the inclination ratio determined by regression analysis. Furthermore, the experiment above was repeated 16 times, and the ESFs from the experiments were overlaid with the least-square method to calculate the synthesized MTF. Using this approach, the accuracy of MTF measurement was improved, even in water, which is more representative of clinical imaging conditions. There was a significant difference between the MTFs in air and water at $1.0 \mathrm{LP} / \mathrm{mm}$, but the MTFs at a higher frequency range (above $2.0 \mathrm{LP} / \mathrm{mm}$ ) were not significantly different. These findings indicate that the accuracy of the measurement needs to be improved.

\section{Acknowledgments}

The authors thank Edanz Group (https://en-author-services.edanzgroup. com/ac) for editing a draft of this manuscript.

\section{Conflict of interest}

Yoshinori Arai has a conflict of interest with J. Morita MFG. Corp.

\section{References}

1. Arai Y, Tammisalo E, Iwai K, Hashimoto K, Shinoda K (1999) Development of a compact computed tomographic apparatus for dental use. Dentomaxillofac Radiol 28, 245-248.

2. Horner K, Islam M, Flygare L, Tsiklakis K, Whaites E (2009) Basic principles for use of dental cone beam computed tomography: consensus guidelines of the European Academy of Dental and Maxillofacial Radiology. Dentomaxillofac Radiol 38, 187-195.

3. Al-Okshi A, Lindh C, Salé H, Gunnarsson M, Rohlin M (2015) Effective dose of cone beam CT (CBCT) of the facial skeleton: a systematic review. Br J Radiol 88, 20140658.

4. Tanimoto H, Arai Y (2009) The effect of voxel size on image reconstruction in cone-beam computed tomography. Oral Radiol 25, 149-153.

5. Suomalainen A, Kiljunen T, Käser Y, Peltola J, Kortesniemi M (2009) Dosimetry and image quality of four dental cone beam computed tomography scanners compared with multislice computed tomography scanners. Dentomaxillofac Radiol 38, 367-378.

6. Watanabe H, Honda E, Kurabayashi T (2010) Modulation transfer function evaluation of cone beam computed tomography for dental use with the oversampling method. Dentomaxillofac Radiol 39, 28-32.

7. Watanabe H, Wagatsuma T, Nomura Y, Honda E, Kurabayashi T (2010) Spatial resolution of FineCube, a newly developed cone-beam computed tomography system. Oral Radiol 26, $56-60$

8. Pauwels R, Stamatakis H, Manousaridis G, Walker A, Michielsen K, Bosmans H et al. (2011) Development and applicability of a quality control phantom for dental cone-beam CT. J Appl Clin Med Phys 12, 245-260.

9. Pauwels R, Beinsberger J, Stamatakis H, Tsiklakis K, Walker A, Bosmans H et al. (2012) Comparison of spatial and contrast resolution for cone-beam computed tomography scanners. Oral Surg Oral Med Oral Pathol Oral Radiol 114, 127-135.

10. Bamba J, Araki K, Endo A, Okano T (2013) Image quality assessment of three cone beam CT machines using the SEDENTEXCT CT phantom. Dentomaxillofac Radiol 42, 1-13.

11. Brüllmann D, Schulze RKW (2014) Spatial resolution in CBCT machines for dental/maxillofacial applications-what do we know today? Dentomaxillofac Radiol 44, 20140204.

12. Elkhateeb SM, Torgersen GR, Arnout EA (2016) Image quality assessment of clinically applied CBCT protocols using a QAT phantom. Dentomaxillofac Radiol 45, 20160075.

13. Dillenseger JP, Gros CI, Sayeh A, Rasamimanana J, Lawniczak F, Leminor JM et al. (2016) Image quality evaluation of small FOV and large FOV CBCT devices for oral and maxillofacial radiology. Dentomaxillofac Radiol 46, 1-10.

14. Oliveira MVLL de, Wenzel A, Campos PSFF, Spin-Neto R (2017) Quality assurance phantoms for cone beam computed tomography: a systematic literature review. Dentomaxillofac Radiol 46, 20160329.

15. Watanabe H, Nomura Y, Kuribayashi A, Kurabayashi T (2018) Spatial resolution measurements by Radia diagnostic software with SEDENTEXCT image quality phantom in cone beam CT for dental use. Dentomaxillofac Radiol 47, 1-6.

16. Schulze RKW, Doering CI (2019) Simple computation of the approximated modulation transfer function (MTF) using spreadsheet-software: method and evaluation in five maxillofacial CBCT-devices. Dentomaxillofac Radiol 48, 20180350.

17. Taylor C (2016) Evaluation of the effects of positioning and configuration on contrastto-noise ratio in the quality control of a 3D Accuitomo 170 dental CBCT system. Dentomaxillofac Radiol 45, 20150430.

18. Buhr E, Günther-Kohfahl S, Neitzel U (2003) Accuracy of a simple method for deriving the presampled modulation transfer function of a digital radiographic system from an edge image. Med Phys 30, 2323-2331.

19. González-López A (2018) Effect of noise on MTF calculations using different phantoms Med Phys 45, 1889-1898.

20. Fujita H, Tsai DY, Itoh T, Doi K, Morishita J, Ueda K et al. (1992) A simple method for determining the modulation transfer function in digital radiography. IEEE Trans Med Imaging 11, 34-39.

21. Nyquist H (1928) Certain topics in telegraph transmission theory. Trans Am Inst Electr Eng 47, 617-644.

22. Ball RL, Barbizam JV, Cohenca N (2013) Intraoperative endodontic applications of conebeam computed tomography. J Endod 39, 548-557.

23. Greer PB, Van Doorn T (2000) Evaluation of an algorithm for the assessment of the MTF using an edge method. Med Phys 27, 2048-2059. 